\title{
Bayesian probability theory to identify false coincidences in coincidence experiments
}

\author{
Pascal Heim ${ }^{1}$, Michael Rumetshofer ${ }^{2}$, Bernhard Thaler $^{1}$, Wolfgang E. Ernst ${ }^{1}$, Wolfgang \\ von der Linden ${ }^{2}$, and Markus Koch ${ }^{1, *}$ \\ 1 Graz University of Technology, Inst. of Experimental Physics, 8010 Graz, Austria \\ 2 Graz University of Technology, Inst. of Theoretical and Computational Physics, 8010 Graz, Austria
}

\begin{abstract}
We describe a Bayesian formalism to analyse femtosecond pump-probe photoionization experiments with photoelectron-photoion coincidence (PEPICO) detection. This approach overcomes the drawback of extraordinary long data acquisition times of PEPICO detection. In extension to simply excluding false coincidences as previously [1], we here present an investigation of their influence on the underlying spectrum. The software is provided at https://github.com/fslab-tugraz/PEPICOBayes/.
\end{abstract}

\section{Introduction}

Coincidence experiments are widely spread and powerful spectroscopic techniques. Recently we applied photoelectron-photoion coincidence (PEPICO) detection to femtosecond photoionization experiments [2]. PEPICO spectra include separated photoelectron spectra for each ion fragment mass and provide additional information compared to sole electron and/or ion detection that can be essential to establish a mechanistic description of the underlying molecular dynamics. An important example are fragmentation studies, where only PEPICO spectra directly provide evidence if molecular fragmentation occurs before ionization in the neutral molecule, or is rather induced by the ionizing pulse and proceeds in the ion [3].

The concept behind PEPICO is to create an electron-ion pair and detect both particles. However, because the actual number of ionization events follows a Poisson probability distribution there is a certain chance for two ionization events caused by a single laser shot. In combination with detection probabilities of electrons and ions less than unity, it can occur that the ion of one molecule and the electron of another molecule are mistaken as a coincidence pair. These situations are referred to as false coincidences, which obscure the PEPICO spectrum. Experimentally, only highly advanced setups are able to identify to some extent false coincidences, which is, however, not possible in conventional, widely used time-of-flight measurements. Experimentalists are therefore forced to work at very low ionization rates well below 0.3 events per laser shot in order to keep the rate of multiple ionization events and thus the rate of false coincidences low [4], often resulting in unacceptable long data acquisition times.

\footnotetext{
*Corresponding author: markus.koch@tugraz.at
} 
Here we present an analysis algorithm, based on Bayesian probability theory to consistently cover this problem. At higher ionization rates the measured PEPICO spectrum is obscured by false coincidences, so that the true spectrum cannot be obtained by the conventional PEPICO method [5]. The Bayesian approach, in contrast, considers the relationship between the true spectrum and the measured PEPICO spectrum, therefore allowing the exclusion of false coincidences from the spectrum with the result that significantly higher ionization rates are acceptably without distortion of the resulting spectrum. While the Bayesian formalism was presented recently in detail [1], we here focus on the role of false coincidences by distinguishing between homo- and heteromolecular false coincidences (see below) and analyse their spectral distribution and how they distort the PEPICO spectrum. We apply the algorithm to PEPICO spectra of acetone molecules.

\section{Methods}

The experiment [2] consists, in brief, of a versatile pump-probe setup based on a commercial Ti:sapphire femtosecond laser system in a typical pump-probe photoionization setup. The kinetic energy of the electrons and charge-to-mass ratio of the ions are measured with a single magnetic bottle type time-of-flight spectrometer.

Table 1. Overview of relevant quantities of the Bayesian method.

\begin{tabular}{|l|c|l|c|l|}
\hline & \multicolumn{2}{|c|}{ spectra } & \multicolumn{2}{c|}{ parameters } \\
\hline $\begin{array}{l}\text { experimentally } \\
\text { accessible }\end{array}$ & $D_{1}$ & $\begin{array}{l}\text { data set 1: pump- } \\
\text { probe, pump-/probe- } \\
\text { only PEPICO spectra }\end{array}$ & $D_{2}$ & $\begin{array}{l}\text { data set 2: count rates of }\left(N_{\mathrm{e}}, N_{\mathrm{i}}\right)- \\
\text { events }\end{array}$ \\
\hline $\begin{array}{l}\text { fundamental } \\
\text { quantities, } \\
\text { experimentally } \\
\text { in-accessible }\end{array}$ & $q_{\beta}$ & $\begin{array}{l}\text { pump-probe spectral } \\
\text { density }\end{array}$ & $\lambda_{\beta}$ & pump-probe ionization rate \\
\cline { 2 - 5 } & $q_{\alpha}$ & $\begin{array}{l}\text { pump-/probe-only } \\
\text { spectral density }\end{array}$ & $\lambda_{\alpha}$ & pump-/probe-only ionization rate \\
\cline { 2 - 5 } & \multicolumn{3}{|c}{$\xi_{e}$} & electron detection probability \\
\cline { 2 - 5 } & $\xi_{i}$ & ion detection probability \\
\hline
\end{tabular}

The principle of the Bayesian method is summarized in Table 1 (details in [1]): Based on experimentally accessible quantities $\left(D_{1}, D_{2}\right)$, the Bayesian formalism estimates fundamental quantities $\left(q_{\alpha}, q_{\beta}, \lambda_{\alpha}, \lambda_{\beta}, \xi_{e}, \xi_{i}\right)$, which fully characterize the investigated processes but are not directly accessible by the experiment. Dataset 1 consists of the measured pump-probe and pump-/probe-only PEPICO background spectra. Dataset 2 consists of detection rates of all possible combinations of $N_{\mathrm{e}}$ electron events and $N_{\mathrm{i}}$ ion events; for example $\left(N_{\mathrm{e}}, N_{\mathrm{i}}\right)=(2,3)$ relates to the number of pump-probe measurements (laser shots) where 2 electrons and 3 ions are detected.

From the fundamental quantities various expectation values, such as covariances and confidence intervals, can be computed. Time-dependent signals are obtained from ionization-rate weighted spectra (e.g., $\lambda_{\beta} q_{\beta}$ ). In terms of false coincidences, it can be distinguished between homo- and heteromolecular false coincidences, where the two involved ionization events create the same and different, respectively, fragments (see equation (26) in [1]). If pump-/probe-only background signals are present, the Bayesian formalism has the following advantages: (1) the signal-to-noise ratio is significantly increased, (2) the background contributions are not overestimated upon subtraction, and (3) prior knowledge, such as positivity of the reconstructed spectrum, is consistently incorporated. 


\section{Results and Discussion}

In the following the Bayesian formalism is applied to PEPICO spectra on acetone molecules, obtained by three photon excitation and one photon ionization [2].

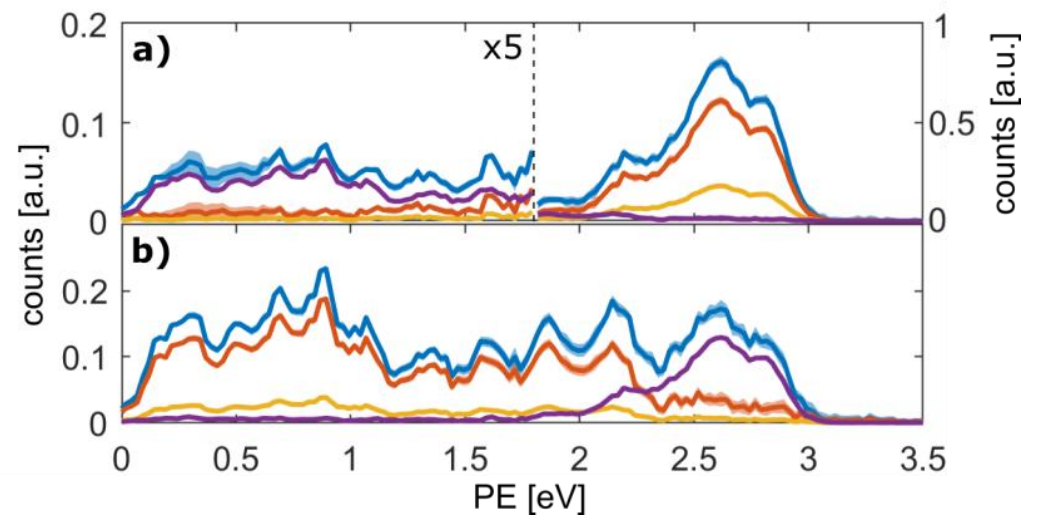

Fig. 1. Bayesian PEPICO spectra associated to acetone (a) and acetyl (b) ions. The total coincidence signal (blue) consists of the true coincidence signal (red), the homo- (yellow) and heteromolecular false coincidence signal (purple). Shaded areas are confidence intervals.

Figure 1 shows PEPICO spectra of acetone parent (a) and acetyl fragment ions (b), recorded at a pump-probe delay of $0 \mathrm{fs}$. The total mean ionization rate $\left(\lambda_{\beta}\right)$ is $(1.15 \pm 0.01)$ with a detection probability of $\xi_{e}=(37.4 \pm 0.2) \%$ and $\xi_{i}=(25.3 \pm 0.2) \%$. At this high ionization rate the influence of false coincidences is clearly visible. In the parent spectrum (a) above $2.2 \mathrm{eV}$ photoelectron energy all false coincidence events are of homomolecular character which mainly originate from two different ionization events both yielding acetone ions (yellow line), and below $2.2 \mathrm{eV}$ most of the false coincidences are of heteromolecular character recorded with an acetyl electron (purple line). Note that the homomolecular false coincidences do not obscure the corresponding electron spectrum, but influence the ratio of different species. Heteromolecular false coincidences, in contrast, obscure the electron spectrum as they have the spectral features of the other species as is clearly seen in the acetone spectrum below $2.2 \mathrm{eV}$ and in the acetyl spectrum above $2.2 \mathrm{eV}$ where most detected coincidences are heteromolecular false coincidences.

In conclusion, we analysed the role of false coincidences in PEPICO spectra by applying Bayesian probability theory. The insight obtained from such analysis can be of importance for identification of the source of false coincidences.

We acknowledge financial support by the Austrian Science Fund (FWF) under grant P29369-N36, as well as support from NAWI Graz.

\section{References}

1. M. Rumetshofer et al., Phys. Rev. A, 97, 062503 (2018)

2. M. Koch, B. Thaler, P. Heim, W. E. Ernst, J. Phys. Chem. A, 121, 6398 (2017)

3. M. Koch, P. Heim, B. Thaler, M. Kitzler, W. E. Ernst, J. Phys. B, 50125102 (2017)

4. V. Stert, W. Radloffa, C. P. Schulz, I. V. Hertel, Eur. Phys. J. D 5, 97 (1999)

5. J. Mikosch, S. Patchkovskii, J. Mod. Optics, 60, 1439 (2013) 